${ }^{1}$ Department of Sport, Culture and the Arts, Strathclyde University, Glasgow G13 1PP

${ }^{2}$ Nursing and Midwifery School, University of Glasgow, Glasgow G12 8LW

${ }^{3}$ Robertson Centre for Biostatistics, University of Glasgow G12 8QQ

${ }^{4} \mathrm{MRC}$ Social and Public Health Sciences Unit, Glasgow G12 8RZ

${ }^{5}$ Cancer Care Research Centre, Department of Nursing and Midwifery, University of Stirling, Stirling FK9 4LA

${ }^{6}$ Beatson Oncology Centre, Glasgow G11 6NT

Correspondence to: N Mutrie nanette.mutrie@strath.ac.uk

doi: 10.1136/bmi.39094.648553.AE

\title{
Benefits of supervised group exercise programme for women being treated for early stage breast cancer: pragmatic randomised controlled trial
}

\author{
Nanette Mutrie, professor of exercise and sport psychology, ${ }^{1}$ Anna M Campbell, research fellow, ${ }^{1}$ Fiona \\ Whyte, Macmillancancerlecturer, ${ }^{2}$ AlexMcConnachie, senioranalyst, ${ }^{3}$ CarolEmslie, researchscientist, ${ }^{4}$ Laura \\ Lee, research assistant, ${ }^{1}$ Nora Kearney, professor of cancer care, ${ }^{5}$ Andrew Walker, health economist, ${ }^{3}$ Diana \\ Ritchie, consultant oncologist ${ }^{6}$
}

\section{ABSTRACT}

Objectives To determine functional and psychological benefits of a 12 week supervised group exercise programme during treatment for early stage breast cancer, with six month follow-up.

Design Pragmatic randomised controlled prospective open trial.

Setting Three National Health Service oncology clinics in Scotland and community exercise facilities.

Participants 203 women entered the study; 177 completed the six month follow-up.

Interventions Supervised 12 week group exercise programme in addition to usual care, compared with usual care.

Main outcome measures Functional assessment of cancer therapy (FACT) questionnaire, Beck depression inventory, positive and negative affect scale, body mass index, seven day recall of physical activity, 12 minute walk test, and assessment of shoulder mobility. Results Mixed effects models with adjustment for baseline values, study site, treatment at baseline, and age gave intervention effect estimates (intervention minus control) at 12 weeks of 129 (95\% confidence interval 83 to 176) for metres walked in 12 minutes, 182 (75 to 289) for minutes of moderate intensity activity reported in a week, 2.6 (1.6 to 3.7) for shoulder mobility, 2.5 (1.0 to 3.9) for breast cancer specific subscale of quality of life, and 4.0 (1.8 to 6.3) for positive mood. No significant effect was seen for general quality of life (FACT$\mathrm{G})$, which was the primary outcome. At the six month follow-up, most of these effects were maintained and an intervention effect for breast cancer specific quality of life emerged. No adverse effects were noted.

Conclusion Supervised group exercise provided functional and psychological benefit after a 12 week intervention and six months later. Clinicians should encourage activity for their patients. Policy makers should consider the inclusion of exercise opportunities in cancer rehabilitation services.

Trial registration Current controlled trials ISRCTN12587864.

\section{INTRODUCTION}

Breast cancer is the most commonly occurring cancer among women in the United Kingdom. More than 40000 new cases are reported each year, and breast cancer accounts for $30 \%$ of the cancer burden in women (excluding non-melanoma skin cancer). Early detection and improved treatments for breast cancer have resulted in increased survival rates; the current five year relative survival rate is estimated to be $80 \%{ }^{12}$ Surviving cancer usually means enduring sequential combinations of treatment modalities (surgery, radiotherapy, systemic chemotherapy, and hormonal treatment). Treatments for cancer can result in significant reductions in many different quality of life outcomes. ${ }^{3}$ Current programmes in cancer rehabilitation are mainly based on psychotherapy or social support. Such therapies do not usually deal with the physical problems encountered by patients, such as fatigue, loss of functional capacity, and weight gain. ${ }^{4}$ Exercise is an intervention that may improve a broad range of quality of life problems after diagnosis of cancer.

Physical activity levels reduce significantly for many women after a diagnosis of breast cancer and remain low after treatment is completed. ${ }^{56} \mathrm{~A}$ prospective observational study in survivors of breast cancer has indicated a 50\% risk reduction in mortality among women who are regularly active compared with those who remained inactive post-diagnosis. ${ }^{7}$ A recent systematic review of the effects of exercise on breast cancer patients and survivors concluded that exercise is an effective intervention to improve quality of life, cardiorespiratory fitness, physical functioning, and fatigue. ${ }^{8}$ However, a more recent Cochrane review, which examined exercise interventions exclusively during treatment for breast cancer, found improvements in physical fitness and activities of daily life but no significant improvements in quality of life or fatigue. ${ }^{9}$ Little of the existing evidence comes from the UK or a National Health Service (NHS) setting.

We aimed to determine if participating in a supervised group exercise programme for women during 
treatment for early stage breast cancer had functional and psychological benefits. We tested the hypotheses that 12 weeks of supervised group exercise, as an adjunct to usual care, would improve quality of life for women during treatment for early stage breast cancer and that benefits would be maintained for six months after the intervention.

\section{METHODS}

\section{Participants}

From January 2004 to January 2005, trained recruiters approached women during appointments at outpatient clinics for chemotherapy or radiotherapy at three NHS oncology centres in Scotland and presented information about the study. ${ }^{10}$ Potential participants with stage 0 -III breast cancer agreed to attend a pre-screening meeting. Exclusion criteria were concurrent unstable cardiac, hypertensive, or respiratory disease; cognitive dysfunction; and regular exercise.

\section{Protocol, assignment, and masking}

This study was a two group (intervention and control) by three time points (baseline, 12 weeks, and six month follow-up) randomised controlled trial. After obtaining written informed consent and baseline measures, we randomly allocated women into one of two groups. The randomisation was stratified by hospital and treatment at baseline (chemotherapy, radiotherapy, or combination) and used randomised permuted blocks of length four and six (that is, for sequences of four or six women in each hospital-treatment combination, exactly half were allocated to each group). Randomisation was done by telephone to an interactive voice response system. We entered and managed all data in an anonymised format; we held data on patient contacts and other administrative data in a separate database. The study was a pragmatic, randomised, prospective, open trial. In exercise studies, blinding the participants to allocation is not possible. We took steps to blind the evaluation of outcomes by having questionnaire responses in sealed envelopes and ensuring that outcome measures were taken by researchers who were not involved in exercise classes. We sent a letter to general practitioners informing them of their patients' participation in the study.

\section{Outcome measures}

The primary outcome measure was quality of life, as measured by the functional assessment of cancer therapy-general (FACT-G) questionnaire. ${ }^{11}$ This questionnaire comprises four core domains of quality of life-physical (FACT-GP), functional (FACT-GF), social (FACT-GS), and emotional (FACT-GE) - and is considered appropriate for use with cancer patients. Breast cancer, fatigue, and endocrine symptoms subscales have been developed and, when added to the FACT-G score, are described as FACT-B, FACT-F, and FACT-ES. ${ }^{1213}$ Secondary outcomes were the Beck depression inventory, the positive and negative affect scale, body mass index, seven day recall of physical activity (Scottish physical activity questionnaire), performance in a 12 minute walk test, and score on a shoulder mobility test. ${ }^{14-18}$

\section{Intervention}

Women assigned to the intervention group received usual care from their healthcare team and, in addition, were invited to attend a supervised group exercise programme. The exercise programme ran for 12 weeks, and women were encouraged to attend two classes and do one additional exercise session at home each week. Fourteen exercise classes led by specifically trained exercise specialists took place in eight community exercise facilities that were all accessible by public transport. Classes were timetabled at various times in the day and evening. The exercise intervention was based on guidelines for prescription of exercise for cancer patients and survivors. ${ }^{19}$ The classes consisted of a warm-up of 5-10 minutes, 20 minutes of exercise (for example walking, cycling, low level aerobics, muscle strengthening exercises, or circuits of specifically tailored exercises), and a cool-down and relaxation period. The exercise class lasted 45 minutes in total. Women were monitored throughout the class to ensure that they were exercising at a moderate level $(50-75 \%$ of age adjusted maximum heart rate). Each week, for six weeks, a specific theme was covered in group discussion after the exercise (for example, the health benefits of exercise, enhancing self efficacy, setting goals) and supported with specifically constructed materials. These themes were guided by a model of behaviour change and were designed to promote independent exercise after the intervention. ${ }^{20} \mathrm{We}$ repeated the six week block on a rolling basis, allowing all participants to hear the same themes. At the end of the 12 week intervention, the women were helped to construct an individual exercise programme and invited to join a local general practice exercise referral scheme. Further details of the intervention and the expertise of the staff are available on our website (www.strath.ac. uk/sca/staff/mutrie_n.html).

Women assigned to the control group received usual care from the healthcare team and completed all outcome measures on the same time frame as the intervention group. After randomisation, this group received a two page leaflet entitled "Exercise after cancer diagnosis," which provided safe guidelines. After the six month follow-up, these women were helped to construct their own personalised exercise plan and invited to join a local general practice exercise referral scheme.

\section{Statistical power and analysis}

With 91 participants in each group, the study was designed to have $90 \%$ power at a $5 \%$ level of significance to detect an intervention effect of approximately 7.5 units on the change in FACT-G score after 12 weeks, assuming a standard deviation of this outcome of 15 units. We based this change on findings from our pilot study. ${ }^{21}$ The primary analysis, and the main analysis applied to each secondary outcome, was to test whether significant differences existed between 
the exercise group and control group in outcomes at the end of the 12 week intervention period and at six months post-intervention, adjusting for the stratification variables (study site and treatment at baseline), age, and baseline value of the outcome. We did the analysis on an intention to treat basis, in the sense that we took no account of adherence to the intervention. We used all available data.

\section{RESULTS}

\section{Participants}

The recruiters approached 1144 women, and 313 agreed to attend pre-screening. We then randomised 203 women. Figure 1 shows the flow of participants through the trial. Table 1 shows the characteristics of the participants. The women in this study were recruited on average six months after diagnosis, had a mean age of just over 50, and came from a range of occupations. No obvious imbalances existed between study groups.

\section{Main outcomes}

Table 2 summarises the outcome variables measured at the baseline, 12 week, and six month post-intervention assessments, for the intervention and control groups separately. Adjusted estimates of the differences between the intervention groups at 12 weeks and six months are also reported. Figure 2 shows effect estimates and confidence intervals for all variables. All effect estimates are reported in units of one standard deviation of the outcome variable in question.
Mixed effects models with adjustment for baseline values, study site, treatment at baseline, and age showed intervention effect estimates (intervention minus control) at 12 weeks of 129 (95\% confidence interval 83 to 176) for metres walked in 12 minutes, 182 (75 to 289) for minutes of moderate intensity activity reported in a week, 2.6 (1.6 to 3.7$)$ for shoulder mobility, 2.5 (1.0 to 3.9) for breast cancer specific subscale quality of life, and 4.0 (1.8 to 6.3) for positive mood. We found no significant intervention effect for FACT-G, which was the primary outcome. We saw non-significant trends towards increases in perceived quality of life in relation to fatigue $(+2.3$ points, $\mathrm{P}=0.091)$ and reduced depression $(-1.7$ points, $\mathrm{P}=0.083)$ in favour of the intervention.

Intervention effect estimates for the six month follow-up data were 105 (60 to 151) for metres walked in 12 minutes, 2.5 (1.4 to 3.6) for shoulder mobility, 1.5 (0.1 to 2.9) for breast cancer specific subscale quality of life (when this subscale is added to the FACT-G a significant effect (4.9, 0.2 to 9.6) for FACT-B emerges), 1.4 (0.3 to 2.5 ) for the social domain (FACT-GS), and 3.9 (1.6 to 6.1) for positive mood. At the follow-up we also saw non-significant trends towards improvements on the primary outcome (FACT-G; +3.6 points, $\mathrm{P}=0.053)$, the functional domain of the FACT-G score (FACT-GF; +1.1 points, $\mathrm{P}=0.067$ ), and depression $(-1.8$ points, $\mathrm{P}=0.064)$ in favour of the intervention.

Figure 3 shows the use of health services in the study up to the six month post-intervention follow-up. Ten

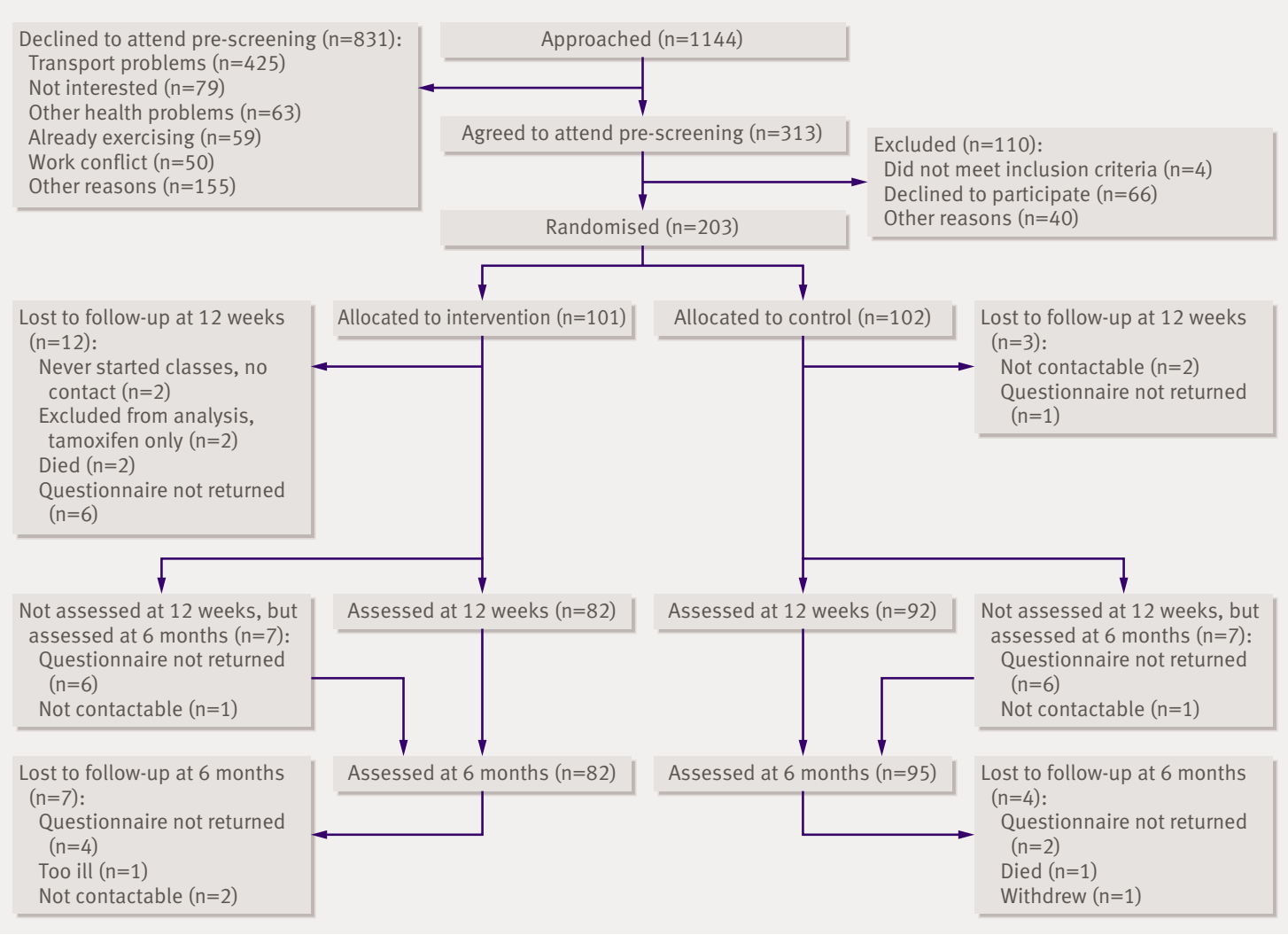




\begin{tabular}{|c|c|c|c|}
\hline Characteristic & All participants $(\mathrm{n}=201)$ & Exercise group $(\mathrm{n}=99)$ & Control group $(n=102)$ \\
\hline Mean (SD) age (years) & $51.6(9.5)$ & $51.3(10.3)$ & $51.8(8.7)$ \\
\hline \multicolumn{4}{|l|}{ Treatment plan: } \\
\hline Chemotherapy & $15(7.5)$ & $8(8.1)$ & $7(6.9)$ \\
\hline Radiotherapy & $57(28.4)$ & $28(28.3)$ & $29(28.4)$ \\
\hline Combination & $129(64.2)$ & $63(63.6)$ & $66(64.7)$ \\
\hline \multicolumn{4}{|l|}{ Study site: } \\
\hline 1 & $33(16.4)$ & $17(17.2)$ & $16(15.7)$ \\
\hline 2 & $151(75.1)$ & $74(74.7)$ & $77(75.5)$ \\
\hline 3 & $17(8.5)$ & $8(8.1)$ & $9(8.8)$ \\
\hline Mean (SD) height (cm) & $160.8(6.1)$ & $160.6(5.9)$ & $161.1(6.3)$ \\
\hline Mean (SD) weight (kg) & $70.8(14.6)$ & $70.2(12.5)$ & $71.5(16.4)$ \\
\hline Mean SD body mass index & $27.4(5.6)$ & $27.3(5.2)$ & $27.5(6.0)$ \\
\hline Mean (SD) days since diagnosis & $162.0(73.8)$ & $162.2(78.0)$ & $161.9(69.8)$ \\
\hline Mastectomy & $81(40.3)$ & $39(39.4)$ & $42(41.2)$ \\
\hline Lumpectomy & $119(59.2)$ & $59(59.6)$ & $60(58.8)$ \\
\hline Reconstructive surgery & $23(11.4)$ & $13(13.1)$ & $10(9.8)$ \\
\hline \multicolumn{4}{|l|}{ Current employment status: } \\
\hline Full/part time & $29(14.4)$ & $16(16.2)$ & $13(12.7)$ \\
\hline Sick & $111(55.2)$ & $49(49.5)$ & $62(60.8)$ \\
\hline Housewife & $26(12.9)$ & $14(14.1)$ & $12(11.8)$ \\
\hline Retired & $35(17.4)$ & $20(20.2)$ & $15(14.7)$ \\
\hline Occupation (before diagnosis): & $(n=171)$ & $(n=82)$ & $(n=89)$ \\
\hline Professional & $48(28.1)$ & $25(30.5)$ & $23(25.8)$ \\
\hline Managerial & $35(20.5)$ & $18(22.0)$ & 17 (19.1) \\
\hline Clerical & $55(32.2)$ & $26(31.7)$ & 29 (32.6) \\
\hline Manual & $33(19.3)$ & $13(15.9)$ & $20(22.5)$ \\
\hline
\end{tabular}

per cent of patients in the intervention group and 20\% in the control group reported at least one night in hospital, and $72 \%$ and $84 \%$ reported at least one visit to their general practitioner. The intervention group reported fewer nights in hospital (Mann-Whitney test, $\mathrm{P}=0.044)$ and fewer visits to their general practitioner $(\mathrm{P}=0.011)$ than the control group.

\section{DISCUSSION}

\section{Principal findings}

Women of all ages (range 29-76 years) and types of occupation were interested in taking part in this study. The main reason for lack of interest was the distance needed for travel to exercise classes. After 12 weeks of supervised exercise, the intervention group showed benefits in physical and psychological

\section{WHAT IS ALREADY KNOWN ON THIS TOPIC}

Exercise has a large potential to improve physical and psychosocial aspects of quality of life in women with breast cancer during and after treatment

Most studies have involved home based or individualised gym based exercise programmes None of the existing evidence comes from the UK or a National Health Service setting

\section{WHAT THIS PAPER ADDS}

Supervised group exercise provided functional and psychological benefits in both the short term and long term for women having treatment for breast cancer

Clinicians should encourage physical activity for patients, and policy makers should consider including exercise opportunities in cancer rehabilitation services functioning in comparison with the control group. No adverse events were reported. The benefits to the intervention group reported at 12 weeks were maintained to the six month follow-up, with the exception of self reported minutes of physical activity. The benefits to breast cancer specific quality of life (FACT-B) from the intervention emerged only at the six month follow-up, when most women were post-treatment. Similar results during breast cancer treatment have been reported, but the exercise intervention was either home based or an individualised gym programme and no follow-up data were reported. ${ }^{22}$

\section{Strengths and weaknesses}

This is the first full scale randomised controlled trial in the UK of a group based exercise programme for breast cancer patients during treatment and has the largest sample size of published exercise trials in breast cancer. The study had an appropriate range and number of participants and is unique in including a follow-up. The dropout rate from the trial was $14 \%$, which is similar to other studies on exercise in cancer. ${ }^{23}$

One weakness is that we do not know which aspect of the group exercise experience provided most benefit. Our qualitative data suggest that the group itself was an important aspect and that exercise in standard settings did not provide the same benefits. ${ }^{24}$ However, studies that have used appropriate comparison groups to rule 


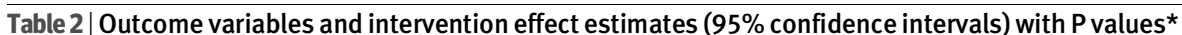

\begin{tabular}{|c|c|c|c|c|c|}
\hline \multirow[b]{2}{*}{ Outcome variable } & \multicolumn{3}{|c|}{ Mean (SD) } & \multicolumn{2}{|c|}{ Effect estimates (exercise-control) } \\
\hline & Baseline & 12 weeks & 6 months & 12 weeks & 6 months \\
\hline \multicolumn{6}{|l|}{ Maximum No: } \\
\hline Control & 102 & 92 & 95 & \multirow[t]{2}{*}{ NA } & \multirow[t]{2}{*}{ NA } \\
\hline Exercise & 99 & 82 & 82 & & \\
\hline \multicolumn{6}{|l|}{ FACT-G: } \\
\hline Control & $73.3(15.0)$ & $77.3(14.4)$ & $77.1(17.0)$ & \multirow[t]{2}{*}{$1.0(-2.7$ to 4.7$) ; P=0.60$} & \multirow[t]{2}{*}{3.6 (0.0 to 7.3$) ; P=0.053$} \\
\hline Exercise & $77.0(12.4)$ & $81.0(16.8)$ & $83.2(12.8)$ & & \\
\hline \multicolumn{6}{|l|}{ FACT-GP: } \\
\hline Control & $20.0(5.7)$ & $21.9(5.1)$ & $22.3(5.3)$ & \multirow{2}{*}{$0.4(-0.8$ to 1.7$) ; \mathrm{P}=0.50$} & \multirow[t]{2}{*}{$0.7(-0.5$ to 2.0$) ; P=0.27$} \\
\hline Exercise & $21.4(4.8)$ & $23.1(4.9)$ & $23.9(4.3)$ & & \\
\hline \multicolumn{6}{|l|}{ FACT-GS: } \\
\hline Control & $23.7(4.8)$ & $23.4(5.0)$ & $22.9(5.5)$ & \multirow[t]{2}{*}{$0.9(-0.2$ to 2.1$) ; P=0.10$} & \multirow[t]{2}{*}{1.4 (0.3 to 2.5$) ; P=0.014$} \\
\hline Exercise & $23.6(5.1)$ & $24.2(4.6)$ & $23.9(4.8)$ & & \\
\hline \multicolumn{6}{|l|}{ FACT-GE: } \\
\hline Control & $18.3(4.7)$ & $18.9(4.4)$ & $18.6(4.5)$ & \multirow{2}{*}{$0.7(-0.3$ to 1.7$) ; P=0.19$} & \multirow[t]{2}{*}{$0.6(-0.4$ to 1.7$) ; P=0.23$} \\
\hline Exercise & $19.0(3.7)$ & $20.1(4.2)$ & $19.7(4.0)$ & & \\
\hline \multicolumn{6}{|l|}{ FACT-GF: } \\
\hline Control & $11.3(5.0)$ & $13.1(5.0)$ & $13.6(5.1)$ & \multirow[t]{2}{*}{$0.4(-0.8$ to 1.6$) ; P=0.49$} & \multirow[t]{2}{*}{$1.1(-0.1$ to 2.3$) ; P=0.067$} \\
\hline Exercise & $12.9(4.7)$ & $14.6(4.6)$ & $15.8(4.2)$ & & \\
\hline FACT-B subscale: & & & & & \\
\hline Control & $21.3(7.0)$ & $22.4(7.2)$ & $24.2(6.3)$ & 2.5 (1.0 to 3.9); $P=0.0007$ & 1.5 (0.1 to 2.9$) ; P=0.039$ \\
\hline Exercise & $22.2(6.7)$ & $25.8(6.0)$ & $26.1(5.6)$ & & \\
\hline FACT-F subscale: & & & & & \\
\hline Control & $32.8(12.7)$ & $36.0(12.1)$ & $37.6(11.8)$ & $2.3(-0.4$ to 5.0$) ; P=0.091$ & $1.9(-0.7$ to 4.6$) ; P=0.15$ \\
\hline Exercise & $36.3(11.7)$ & $40.3(10.4)$ & $41.3(9.7)$ & & \\
\hline FACT-ES subscale: & & & & & \\
\hline Control & $39.9(9.3)$ & $40.3(9.7)$ & $39.7(10.2)$ & $1.1(-1.2$ to 3.4$) ; P=0.36$ & $1.1(-1.2$ to 3.4$) ; P=0.35$ \\
\hline Exercise & $40.6(9.6)$ & $41.6(9.1)$ & $41.0(9.8)$ & & \\
\hline BDI score: & & & & & \\
\hline Control & $13.0(7.4)$ & $11.5(8.6)$ & $10.8(7.5)$ & $-1.7(-3.7$ to 0.2$) ; P=0.083$ & $-1.8(-3.8$ to 0.1$) ; P=0.064$ \\
\hline Exercise & $11.8(6.9)$ & $8.6(6.8)$ & $8.4(7.2)$ & & \\
\hline PANAS positive: & & & & & \\
\hline Control & $28.0(9.2)$ & $29.3(9.8)$ & $29.2(10.5)$ & 4.0 (1.8 to 6.3 ); $P=0.0005$ & 3.9 (1.6 to 6.1); $P=0.0008$ \\
\hline Exercise & $27.7(8.4)$ & $33.4(8.5)$ & $33.0(8.1)$ & & \\
\hline PANAS negative: & & & & & \\
\hline Control & $19.1(7.7)$ & $17.7(7.4)$ & $17.4(6.9)$ & $-0.7(-2.5$ to 1.0$) ; P=0.41$ & $-0.7(-2.5$ to 1.0$) ; P=0.39$ \\
\hline Exercise & $17.3(6.9)$ & $15.6(6.6)$ & $15.7(6.1)$ & & \\
\hline 12 minute walk $(\mathrm{m})$ : & & & & & \\
\hline Control & $975(235)$ & $984(221)$ & $1013(190)$ & 129 (83 to 176); $P<0.0001$ & 105 (60 to 151); P<0.0001 \\
\hline Exercise & $997(211)$ & $1135(143)$ & $1127(166)$ & & \\
\hline SPAQ leisure activity & & & & & \\
\hline Control & $365(288)$ & $416(405)$ & $427(370)$ & 182 (75 to 289 ); $P=0.0009$ & 64 (-41 to 169$) ; P=0.23$ \\
\hline Exercise & $367(306)$ & $585(385)$ & $492(327)$ & & \\
\hline Shoulder mobility sc & & & & & \\
\hline Control & $30.5(5.6)$ & $30.1(5.9)$ & $29.6(6.2)$ & 2.6 (1.6 to 3.7); P<0.0001 & 2.5 (1.4 to 3.6); P<0.0001 \\
\hline Exercise & $31.1(5.4)$ & $33.2(4.6)$ & $32.8(4.8)$ & & \\
\hline Body mass index: & & & & & \\
\hline Control & $27.5(6.0)$ & $27.9(6.9)$ & $27.0(5.4)$ & $-0.5(-1.3$ to 0.2$) ; P=0.16$ & $-0.2(-0.9$ to 0.5$) ; P=0.59$ \\
\hline Exercise & $27.3(5.2)$ & $26.9(4.3)$ & $27.0(4.6)$ & & \\
\hline
\end{tabular}

$\mathrm{BDI}=$ Beck depression inventory; $\mathrm{FACT}=$ functional assessment of cancer therapy (see text for core domains and subscales); NA=not applicable; $\mathrm{PANAS}=$ positive and negative affect scale; $\mathrm{SPAQ}=\mathrm{Scottish}$ physical activity questionnaire.

*Based on mixed effects models with adjustment for baseline values, study site, treatment at baseline, and age.

out a placebo effect suggest that these beneficial effects cannot be completely attributed to non-specific characteristics of the programme (such as increased attention from fitness instructors) or support received from fellow patients. ${ }^{2225}$ In addition, improvements in the 12 minute walk and shoulder mobility tests in favour of the intervention group are more directly attributable to the exercise than to the group effect. 


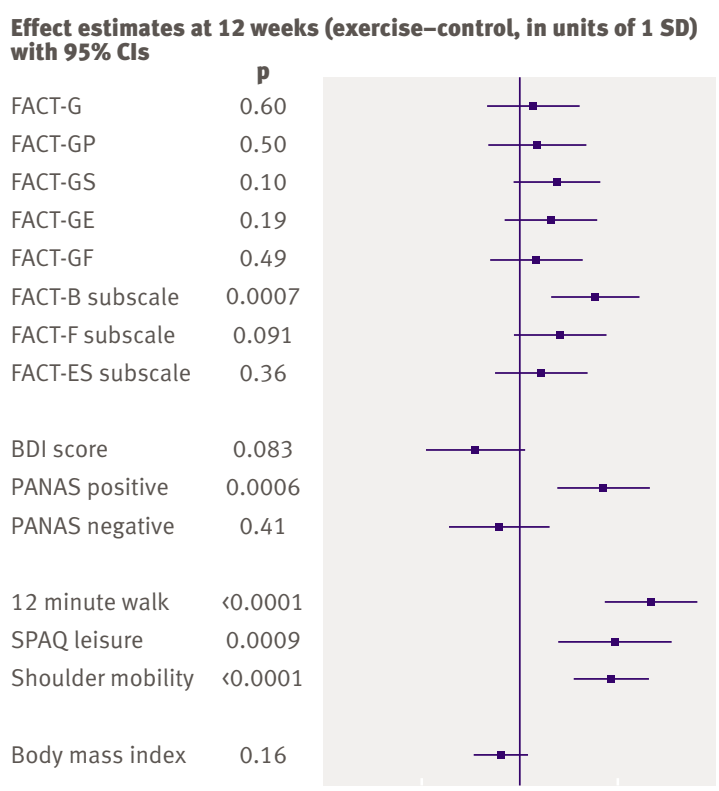

Effect estimates at 6 months (exercise-control, in units of 1 SD) with $95 \%$ Cls

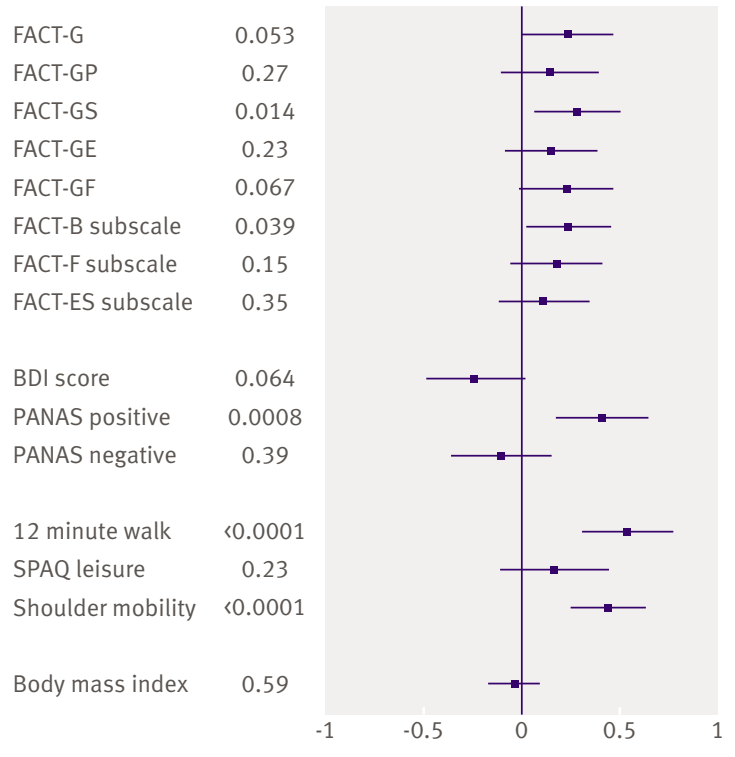

Fig 2 | Effect estimates (intervention minus control), with $95 \%$ confidence intervals and $P$ values, for outcome variables at the 12 week assessment (top) and the six month follow-up assessment (bottom), expressed in units of one standard deviation (SD) of the outcome distributions, based on mixed effects models with adjustment for baseline values, study site, treatment at baseline, and age. BDI=Beck depression inventory; FACT=functional assessment of cancer therapy (see text for core domains and subscales); PANAS=positive and negative affect scale; SPAQ=Scottish physical activity questionnaire

\section{Meaning}

A diagnosis of cancer can signal a "teachable moment," and patients often show an enhanced motivation to change lifestyle behaviours. ${ }^{26}$ Women benefited from the provision of exercise classes, and these benefits may be caused by the exercise itself, by the group experience, or by a combination of both. Clinicians

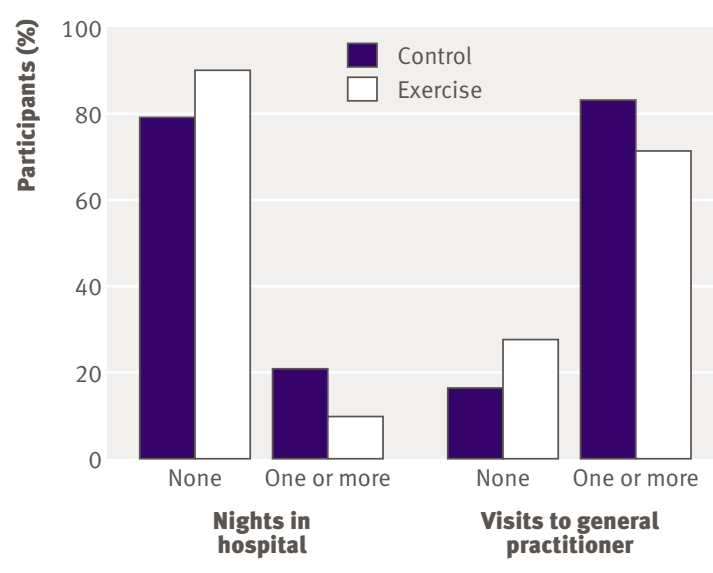

Fig 3 | Percentages of patients in the control and intervention groups who reported at least one night in hospital or at leas one visit to their general practitioner during the study

should encourage activity for patients with cancer, and policy makers should consider including opportunities for exercise in cancer rehabilitation services, similar to the exercise component in cardiac rehabilitation. Further research is needed on the effects of exercise on patients with and survivors of cancers other than breast cancer. In addition, home based programmes need to be evaluated, as many women could not attend exercise classes because of the distance they needed to travel; this would also allow a test of exercise alone without the group effect.

\section{Conclusion}

Supervised group exercise in addition to usual care for women receiving treatment for early stage breast cancer provided functional and psychological benefit at the end of a 12 week programme and at the six month follow-up.

Contributors: NM, AMC, NK, FW, and Gaye Patterson planned the project. All authors supervised the conduct of the study. AMC, Fiona Scott, and Claire Wilson taught the exercise classes. AMC, LL, Fiona Scott, Claire Wilson, and CE collected the data. FW, DR, and NK provided clinical expertise. CE was responsible for qualitative aspects. AMcC was responsible for data handling and analysis. Mary Smith, Janice Japp, and Linda Robb recruited the participants. NM and $\mathrm{AMC}$ wrote the first draft, and all authors contributed to the final paper and agreed revisions. NM is the guarantor

Funding: Cancer Research UK. The funders were independent from the conduct and outcomes of this study. CE is funded by the UK Medical Research Council.

Competing interests: None declared.

Ethical approval: West ethics committee of Greater Glasgow Health Board (LREC Ref:03/22(2)).

1 Cancer Research UK. Breast cancer survival statistics 2005: info. cancerresearchuk.org/cancerstats/types/breast/survival.

2 Coleman M, Rachet B, Woods L, Mitry E, Riga M, Cooper N, et al. Trends and socioeconomic inequalities in cancer survival in England and Wales up to 2001. Br J Cancer 2004;90:1367-73.

3 Courneya KS, Friedenreich CM. Physical exercise and quality of life following cancer diagnosis: a literature review. Annals of Behavioural Medicine 1999;21:171-9.

4 Wilmoth MC, Coleman EA, Smith SC, Davis C. Fatigue, weight gain, and altered sexuality in patients with breast cancer: exploration of symptom cluster. Oncol Nurs Forum 2004;31:1069-75.

5 Irwin M, Crumley D, McTiernan A, Bernstein L, Baumgartner R, Gilliland F, et al. Physical activity levels before and after a diagnosis of breast carcinoma. Cancer 2003;97:1746-57. 
6 Irwin M, McTiernan A, Bernstein L, Gilliland F, Baumgartner R, Baumgartner K, et al. Physical activity levels among breast cancer survivors. Med Sci Sports Exerc 2004;36:1484-91.

7 Holmes MD, Chen WY, Feskanich D, Kroenke CH, Colditz GA. Physical activity and survival after breast cancer diagnosis. JAMA 2005;293:2479-86.

8 McNeely ML, Campbell KL, Rowe BH, Klassen TP, Mackey JR, Courneya KS. Effects of exercise on breast cancer patients and survivors: a systematic review and meta-analysis. CMAJ 2006;175:34-41.

9 Markes M, Brockow T, Resch K. Exercise for women receiving adjuvant therapy for breast cancer. Cochrane Database Syst Rev 2006;(4):CD005001.

10 Campbell A, Whyte F, Mutrie N. Strategies to improve recruitment to an exercise intervention during breast cancer treatment. Clinical Effectiveness in Nursing 2007;9:211-3.

11 Cella DF, Tulsky DS, Gray G, Sarafian B, Linn E, Bonomi A, et al. The functional assessment of cancer therapy scale: development and validation of the general measure. J Clin Oncol 1993;11:570-9.

12 Brady MJ, Cella DF, Mo F, Bonomi AE, Tulsky DS, Lloyd SR, et al. Reliability and validity of the functional assessment of cancer therapy—breast quality-of-life instrument. J Clin Oncol 1997;15:974-86.

13 Fallowfield LI, Leaity SK, Howell A, Benson S, Cella D. Assessment of quality of life in women undergoing hormonal therapy for breast cancer: validation of an endocrine symptom subscale for the FACT-B. Breast Cancer Res Treat 1999;55:187-97.

14 Beck AT, Steer RA, Brown GK. Manual for the Beck depression inventory-2. San Antonio, Texas: Psychological Corporation, 1996.

15 Watson D, Clark L, Tellegen A. Development and validation of brief measures of positive and negative effect: the PANAS scales. J Pers Soc Psychol 1988;54:1063-70.

16 Lowther M, Mutrie N, Loughlan C, McFarlane C. Development of a Scottish physical activity questionnaire: a tool for use in physical activity interventions. Br J Sports Med 1999;33:1-6.
17 McGavin CR, Gupta SP, McHardy GJR. Twelve-minute walking test for assessing disability in chronic bronchitis. BMJ 1976;i:822-3.

18 Halverstadt A, Leonard A. Essential exercises for breast cancer survivors. Boston Massachusetts: Harvard Common Press, 2000.

19 Courneya KS, Mackey JR, McKenzie D. Exercise for breast cancer survivors: research evidence and clinical guidelines. Phys Sportsmed 2002;30(8):33-42.

20 Marcus B, Eaton C, Rossi JS, Harlow L. Self-efficacy, decision-making, and stages of change: an integrative model of physical exercise. J Appl Soc Psychol 1994;24:489-508.

21 Campbell A, Mutrie N, White F, McGuire F, Kearney N. A pilot study of a supervised group exercise programme as a rehabilitation treatment for women with breast cancer receiving adjuvant treatment. Eur J Oncol Nurs 2005;9:56-63.

22 Segal R, Evans W, Johnson D, Smith J, Colletta S, Gayton J, et al. Structured exercise improves physical functioning in women with stages I and II breast cancer: results of a randomized controlled trial. J Clin Oncol 2001;19:657-65.

23 Oldervoll LM, Kaasa S, Hjermstad MJ, Lund JA, Loge JH. Physical exercise results in the improved subjective well-being of a few or is effective rehabilitation for all cancer patients? Eur J Cancer 2004;40:951-62.

24 Emslie C, Whyte F, Campbell A, Mutrie N, Lee L, Ritchie D, et al. “ wouldn't have been interested in just sitting round a table talking about cancer"; exploring the experiences of women with breast cancer in a group exercise trial. Health Educ Res (in press).

25 Courneya KS, Friedenreich C, Sela R, Quinney A, Rhodes R, Handman M. The group psychotherapy and home-based physical exercise (GROUP-HOPE) trial in cancer survivors: physical fitness and quality of life outcomes. Psycho-Oncology 2003;12:357-74.

26 Demark-Wahnefried W, Aziz NM, Rowland JH, Pinto BM. Riding the crest of the teachable moment: promoting long-term health after the diagnosis of cancer. I Clin Oncol 2005;23:5814-30.

Accepted: 26 December 2006 